\title{
Magnitude estimates for electrical pulses: Evidence for two neural mechanisms
}

\author{
ATSUKI HIGASHIYAMA \\ University of Osaka Prefecture, Osaka, Japan \\ and \\ TAKARA TASHIRO \\ Osaka City University, Osaka, Japan
}

\begin{abstract}
The hypothesis that there are two neural mechanisms for electrocutaneous stimulation-one that is sensitive to low current and is adaptive to repeated stimulation and another that is responsive to high current and is less adaptive-was tested in a control and four main experiments. In the main experiments, magnitude estimates obtained for single electrical pulses (of 2-msec duration) were described by a simple power function for each combination of high- and low-current levels and 10 trial blocks. The results were: (1) The slope of the power function for low current was steeper than was that for high current; (2) for low current, the intercept of the power function decreased with increasing block, whereas for high current, it remained constant over blocks; (3) this decrease of the intercept for low current disappeared when judgmental blocks were separated by a rest period of $8 \mathrm{~min}$; (4) the modulus did not affect the slope; (5) for a large modulus combined with low current, the intercept decreased rapidly over trial blocks, whereas for a small modulus combined with high current, the intercept increased over trial blocks. The first four findings support the two-mechanism hypothesis, but the last one may also be interpretable in terms of the regression to absolute scale values.
\end{abstract}

Higashiyama and Tashiro (1987) obtained free-modulus magnitude estimates for single electrical pulses as a function of stimulus current, with the parameters of trial block and current level. For the high-current level, which produced pain responses, the magnitude estimates remained constant independently of block, whereas for the low-current level, which yielded tactile sensations, they decreased as a function of block. In addition, the exponents of power functions fitted to the magnitude estimates were smaller for the high-current level (2.02) than were those for the low-current level (4.06). These findings have led them to suggest that there are two neural mechanisms with different adaptation processes: One is sensitive to low current and is very adaptive to repeated stimulation, and another is responsive to high current and is less adaptive.

However, these outcomes also could be explained in terms of a natural absolute scale. Zwislocki and Goodman (1980) argued that subjects tend to use absolute judgments rather than ratio judgments when making magnitude estimations or productions of sensation. In usual ratio scales, the unit of measurement is arbitrary and can be changed by multiplying all scale values by a constant. In absolute scales, on the other hand, the unit is not arbitrary

This study was supported by Grant-in-Aids for Scientific Research (Nos. 61510054 and 63710068) provided by the Japanese Ministry of Education, Science, and Culture. Correspondence may be sent to Atsuki Higashiyama, Psychology Laboratory, University of Osaka Prefecture, Mozu-umemachi, Sakai, Osaka 591, Japan. and therefore cannot be changed. In other words, an absolute scale assumes a rigid correspondence between numerals and subjective magnitude (Gescheider, 1985). It is possible that low-current subjects start by assigning arbitrary numbers larger than the absolute scale values, and that, as these subjects become more familiar with the electrical pulses, they begin to make smaller estimates that more closely correspond to those values. However, for high-current subjects, this effect would probably be obscured due to the fact that their initial estimates would closely correspond to the absolute scale values.

This study was designed to provide stronger conclusions about the postulated neural mechanisms. In Experiment 1, we repeated our previous study (Higashiyama \& Tashiro, 1987): Subjects made free-modulus magnitude estimations for six current values at both high- and low-current levels. The high-current level ranged from 2.8 to 3.8 times as intense as the threshold current; the low-current level ranged from 1.3 to 1.8 times as intense.

In Experiment 2, the effects of modulus were examined by assigning the number 1 or 100 to the first presented current for each current level. Under the two-neuralmechanism hypothesis, no effect of modulus is expected, because neural mechanisms of cutaneous sensations and pain are generally independent of the subjective number system. On the other hand, if the modulus assigned to a current value is greatly different from the corresponding absolute scale value, an effect of modulus may be expected, because estimates will change in the direction of the absolute scale value as judgments are repeated. 
In Experiment 3, we examined the effects of repetition of electrical pulses on magnitude estimates by inserting an 8-min rest period between judgmental blocks. If the decrease in judged magnitude with repetition of lowcurrent electrical pulses were due to sensory adaptation, judgments would stay the same or increase from immediately before to immediately after the rest period. This hypothesis is based on the assumption that the cutaneous sensitivity to electrical stimulation holds steady or recovers from adaptation during a rest period; certainly it does not decrease in the absence of stimulation. On the other hand, if the estimates are regressing to absolute scale values, postjudgments would be smaller than prejudgments, since that regression is unlikely to stop during the rest period.

In Experiment 4, the subjects judged over the whole current range (current ratios of 1.3 to 3.8 ), including the high- and low-current levels. If there was no interaction between the two neural mechanisms, the results of Experiment 4 would be identical to those of Experiments 1 and 2.

In Experiment 5, the subjects made magnitude estimations for apparent distance in an outdoor setting. Since the dynamic range, neural process, and adaptability for visual distance differed from those for electrical stimulation, Experiment 5 provided a control situation. If the results of Experiments 1-4 were in agreement with those of Experiment 5, they might be attributed to the judgmental strategies that are common to both sense modalities. Conversely, if different outcomes were obtained, they might be interpreted as the differences of neural mechanisms that regulate the modalities in their own ways.

\section{GENERAL METHOD}

\section{Subjects}

The subjects for electrical stimulation (Experiments 1-4) were a total of 64 undergraduates; their ages ranged from 18 to 22 years. They were paid to participate in a 1-h session (Experiment 4) or in two sessions separated by a day or more (Experiments 1-3). The subjects for visual distance (Experiment 5) were 20 volunteers from the university community; their ages ranged from 18 to 22 years. All subjects for electrical stimulation were advised that the experiment might include painful stimuli and that they were free to withdraw participation at any time during the experiment.

\section{Apparatus}

A Nihonkoden SEN-7203 electric stimulator provided the basic pulse-forming and time circuit. It was used with a Nihonkoden SS$102 \mathrm{~J}$ isolator, which amplified the basic current pulse delivered from the stimulator and provided a constant current pulse of controllable amplitude by adjusting a variable resistor in the stimulator. Higashiyama and Tashiro (1988) provided tracings of oscilloscopic waveforms that were measured at the output of the isolator. The combination system of an Apple II microcomputer and a Sanwa time regulator determined the time schedule of warning buzzer, foreperiod, and intertrial interval. Pulse shape and duration were calibrated with a Hitachi Type V-302 oscilloscope; pulse amplitude was measured by a Sanwa Electronic Instrument Model 9000EA digital ammeter.

A pair of rectangular silver electrodes were constructed to fit into a plastic plate (1 cm wide $\times 2.9 \mathrm{~cm}$ long) that was strapped to the underside of the subject's right wrist in the vicinity of the ulnar nerve. The size of each electrode was $1 \times .7 \mathrm{~cm}$; the longer side of an electrode faced that of another electrode, with the inner edges separated laterally by a distance of $1.5 \mathrm{~cm}$ (for details of electrodes, see Higashiyama \& Tashiro, 1983).

\section{Procedure}

The subject was seated, with his/her right hand resting on a table. Prior to attachment of the electrodes, the subject's wrist was washed with an alcohol solution. The electrodes were then filled with keratin electrode paste and applied to the underside of wrist, with the cathode close to the elbow and the anode close to the palm. A trial sequence was started with a warning buzzer, followed by a 2-msec pulse to electrodes after a foreperiod of $2.16,3.47$, or $4.81 \mathrm{sec}$. Foreperiod was randomized for each trial. Intertrial interval was approximately $10 \mathrm{sec}$.

Using a staircase procedure, we determined a threshold current for each subject. The subject was asked to make more than 32 yes/no judgments of whether current was present on the skin. If current was detected, it was decreased by a step; if it was not, it was increased by a step. The step size of current was about $.08 \mathrm{~mA}$. A pulse was presented on every trial; no blank trials were used. The time schedule of warning buzzer, foreperiod, and intertrial interval was identical with that used in determining threshold.

Immediately after the yes/no judgments were completed, the experimenter estimated the threshold current, in accordance with the computational procedure of Dixon and Massey (1957), and then determined the individual stimulus current values used in magnitude estimation. For the low-current level, the ratios of stimulus current to the threshold current were $1.3,1.4,1.5,1.6,1.7$, and 1.8; for the high-current level, they were $2.8,3.0,3.2,3.4,3.6$, and 3.8; and for the whole range, they were $1.3,1.5,1.7,1.9$, $2.2,2.5,2.8,3.3$, and 3.8. Each stimulus was presented 10 times in randomized order, with the restriction that a block included six different current values for the high- or low-current condition and nine different values for the whole range condition.

\section{EXPERIMENT 1}

Twelve subjects made magnitude estimations without modulus or standard. Half of the subjects took part first in the low-current condition and then in the high-current condition; the remaining subjects took part in the reverse order.

\section{Results and Discussion}

Figure 1 shows the results of Experiment 1. The ordinate represents magnitude estimates on a log scale, and the abscissa represents ratio of stimulus current to threshold current on a log scale. The parameters are trial block and current level. Each data point is a geometric mean taken across the 12 subjects.

Magnitude estimates. A three-way (level $\times$ stimulus $x$ block) ANOVA for repeated measures was performed on the logarithmic transformations of magnitude estimates. The results showed that the main effects of level $[F(1,11)=28.4, p<.001]$, stimulus $[F(5,55)=91.3$, $p<.001]$, and block $[F(9,99)=5.7, p<.001]$ were significant.

The level $\times$ stimulus interaction was significant $[F(5,55)=10.5, p<.001]$, indicating that the magnitude estimates for the low-current level grew more rapidly 


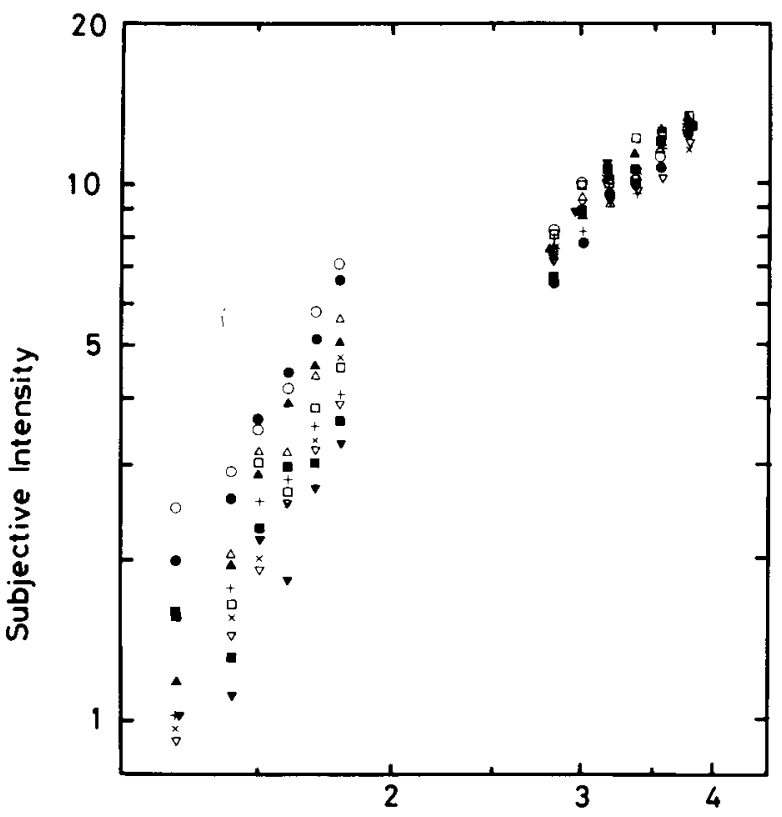

Ratio of Stimulus to Threshold

Figure 1. Log magnitude estimate as a function of $\log$ ratio of stimulus current to threshold current. Each point is the geometric mean of 12 magnitude estimates. The parameter is block: $0=1$ st; $\bullet=$ 2nd; $\Delta=3 \mathrm{rd}$; $\Delta=4$ th; $\square=5$ th; $\equiv=6$ th; $+=7$ th; $x=$ 8th; $\nabla=9$ th; $\nabla=10$ th.

as a function of stimulus current than did those for the high-current level.

The level $\times$ block interaction also was significant $[F(9,99)=7.3, p<.001]$. This suggests that the magnitude estimates generally decreased as a function of block for the low-current level, whereas they remained constant for the high-current level. This decrease of magnitude estimates for the low-current level may be similar to what is called the modulus drop. Ward (1973) found that when a modulus of 10 was assigned to the first presented stimulus in the magnitude estimation of loudness, the estimates for this stimulus decreased over trials and became less than 2 at the 50th trial. Ward interpreted the modulus drop as an accumulated effect of a negative time-order error (Hellström, 1985), which means that with two successive stimuli that are physically equal, the second stimulus is consistently judged to be smaller than the first.

Slopes and intercepts. The magnitude estimates $(\psi)$ were represented as a power function of the ratio of stimulus current $(\phi)$ to threshold current $\left(\phi_{0}\right)$ :

$$
\psi=k\left(\phi / \phi_{0}\right)^{n},
$$

where $k$ and $n$ are constants. According to a least squares criterion, the slope $(n)$ and intercept $(\log k)$ were individually obtained for each combination of block and current level.

The left and right panels of Figure 2 show mean slopes and mean intercepts as a function of block for each current level, respectively. Separate two-way (level $\times$ block) ANOVAs for repeated measures were performed on the slope and intercept data. For the slope data, the main effect of level was significant $[F(1,11)=11.4, p<.01]$, and the level $\times$ block interaction was significant $[F(9,99)$ $=2.1, p<.05]$. An analysis of simple main effects revealed that there was a significant effect of block at the low-current level $[F(9,198)=2.9, p<.01]$, but not at the high-current level. Thus, this interaction may be due to the fact that slopes obtained for the low-current level fluctuated, whereas slopes obtained for the high-current level were constant.

For the intercept data, the level $\times$ block interaction was significant $[F(9,99)=2.4, p<.05]$. An analysis of sim-
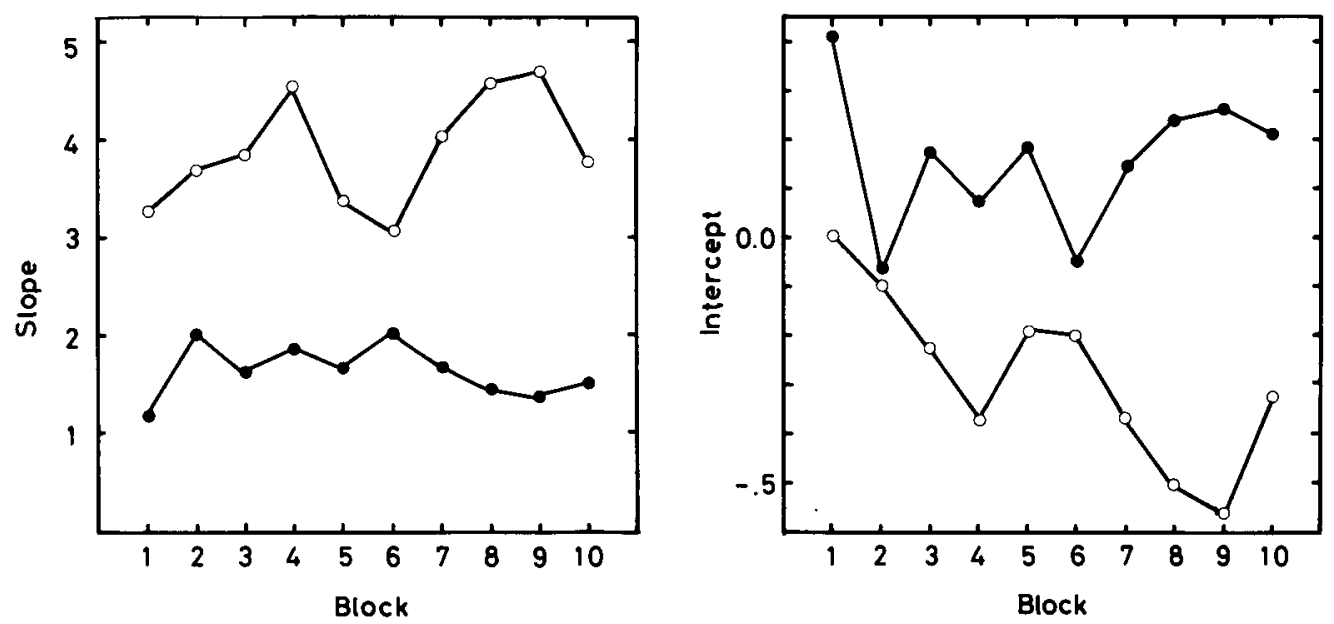

Figure 2. Slope (left) and intercept (right) of power functions fitted to magnitude estimate as a function of block. The parameter is current level: $0=$ low-current level; $\bullet=$ high-current level. 
ple main effects revealed that there was a significant effect of block at the low-current level $[F(9,198)=2.5$, $p<.05$, but not at the high-current level. An analysis of trend of this interaction, furthermore, showed that the linear $[F(1,22)=3.7, .05<p<.10]$, quadratic $(F=1.0)$, and cubic $(F<1)$ components were not significant, but that the linear component accounted for $49 \%$ of the variance.

These results suggest that (1) the exponents of power functions fitted to magnitude estimates were larger for the low-current level $(m=3.9)$ than they were for the highcurrent level $(m=1.6)$ and (2) the magnitude estimates generally decreased with increasing block for the lowcurrent level, whereas they remained constant over blocks for the high-current level. These findings agree with previous results (Higashiyama \& Tashiro, 1987).

\section{EXPERIMENT 2}

In Experiment 2, we examined whether the trend of decreasing magnitude estimates with increasing block is due to the regression of magnitude estimates to absolute scale values. One experienced and 19 naive undergraduates were employed as the subjects. Two groups (each with 10 subjects) followed the same procedure as that used in Experiment 1, except that, for the low-modulus group, the number 1 was assigned to the current presented first, and for the high-modulus group, the number 100. The $\phi / \phi_{0}$ values of the first current were 1.5 for the lowcurrent level and 3.2 for the high-current level. After ensuring the correspondence between the first current and the prescribed number, the experimenter delivered a current that was selected randomly for the first trial of magnitude estimation and asked the subject to represent the subjective intensity relative to the prescribed modulus. This procedure was repeated on subsequent trials without another presentation of the modulus. Half of each modulus group took part first in the low-current level and then in the high-current level; the remaining subjects took part in the reverse order.

If magnitude estimates were to regress to absolute scale values, the low-modulus subjects would make larger estimates as they were experiencing the high-current pulses, whereas the high-modulus subjects would make smaller estimates as they were becoming familiar with the lowcurrent pulses. On the other hand, if adaptation were to occur at the low-current level, the magnitude estimates would decrease with increasing block, regardless of the size of modulus.

\section{Results}

Figure 3 shows the results of the low- and high-current groups, respectively. In both panels of Figure 3, the geometric mean of magnitude estimates is represented as a function of $\phi / \phi_{0}$, with the parameters of level and block.

Magnitude estimates. Separate three-way (level $x$ stimulus $\times$ block) ANOVAs for repeated measures were

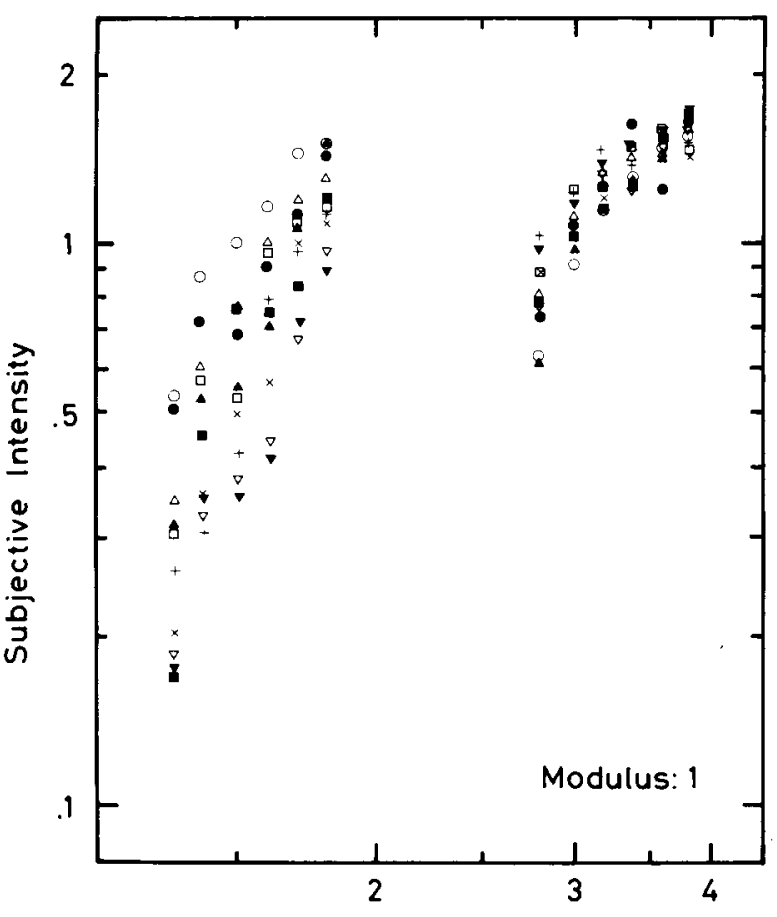

Ratio of Stimulus to Threshold

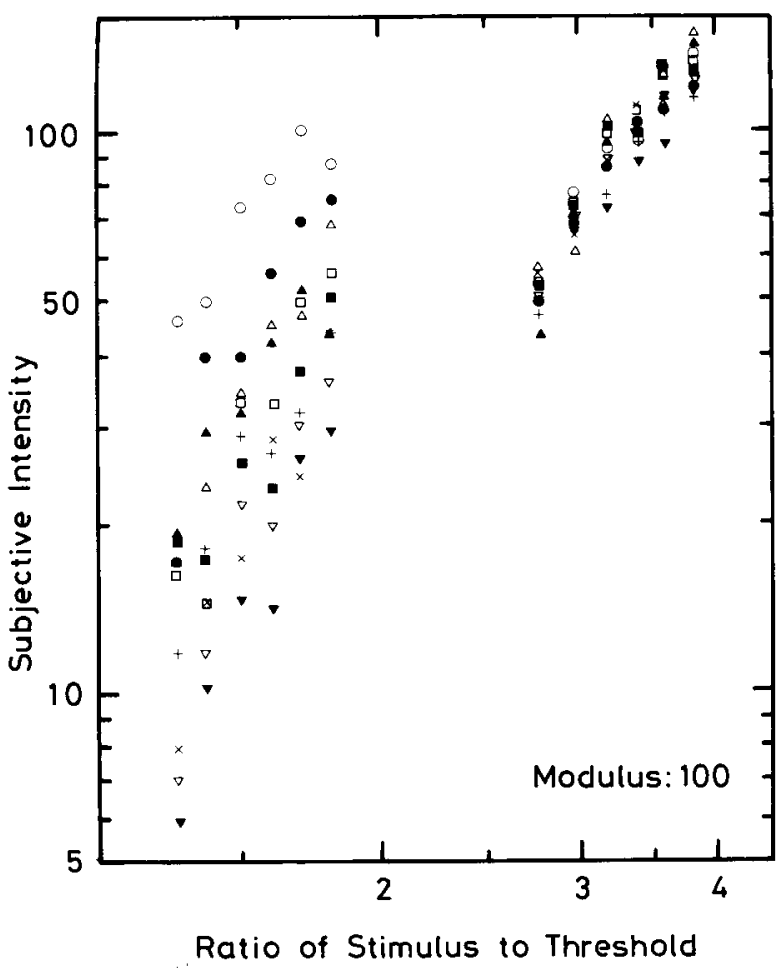

Figure 3. $\mathrm{Log}$ magnitude estimate as a function of $\log$ ratio of stimulus current to threshold current with block and modulus as parameters. Each point is the geometric mean of 10 magnitude estimates. Blocks are indicated by these symbols: $\circ=1$ st; $\bullet=2$ nd; $\Delta=3 \mathrm{rd} ; \Delta=4 \mathrm{th} ; \square=5$ th; $\square=6$ th; $+=7$ th; $x=8$ th; $\nabla=$ 9th; $\nabla=10$ th. 

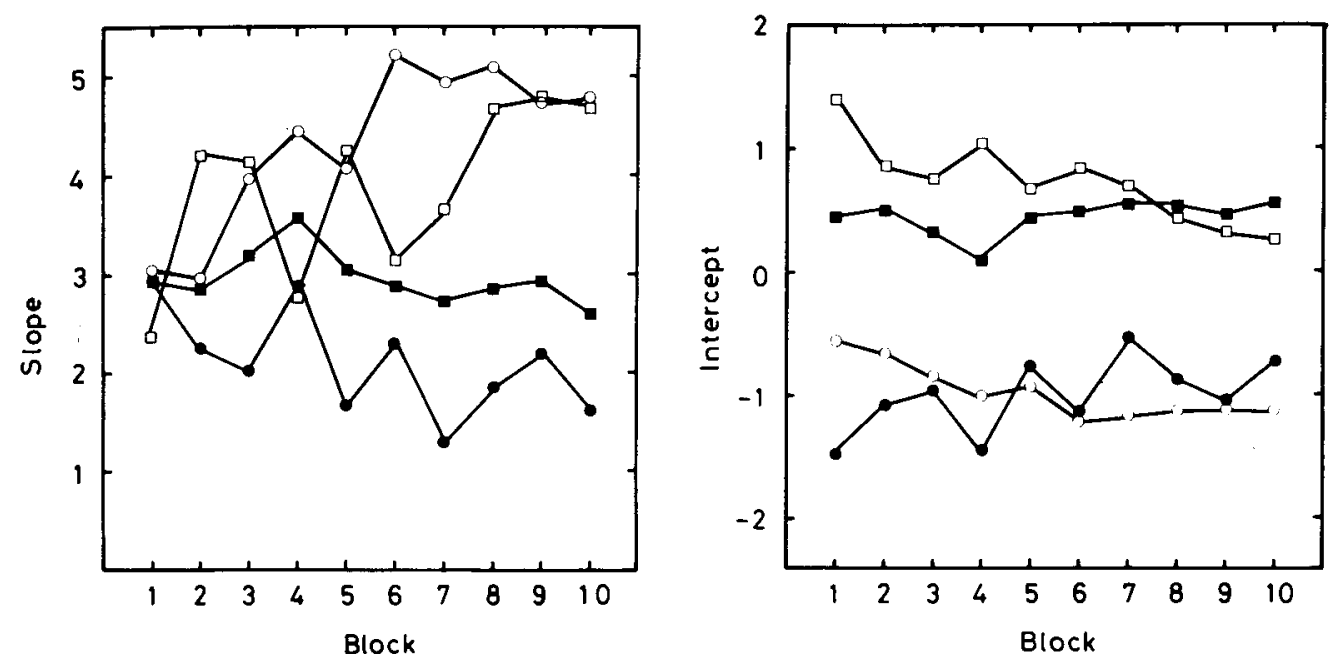

Figure 4. Slope (left) and intercept (right) of power functions fitted to magnitude estimate as a function of block. Parameters are current level and modulus size: Open symbols stand for low-current level and filled symbols for high-current level; circles stand for the modulus of 1 and squares for the modulus of 100 .

performed on the logarithmic transformations of magnitude estimates for the low- and high-modulus groups. For the low-modulus group, there were significant main effects of level $[F(1,9)=7.7, p<.001]$, stimulus $[F(5,45)$ $=33.4, p<.001]$, and block $[F(9,81)=6.1, p<$ $.001]$. Also, there were two significant interactions of level $\times$ stimulus $[F(5,45)=9.5, p<.001]$ and level $\times$ block $[F(9,81)=6.9, p<.001]$.

For the high-modulus group, there were significant main effects of level $[F(1,9)=21.4, p<.001]$, stimulus $[F(5,45)=29.3, p<.001]$, and block $[F(9,81)=$ $6.6, p<.001]$. The level $\times$ block interaction was also significant $[F(9,81)=7.5, p<.001]$, but the level $\times$ stimulus interaction was not significant.

Slope and intercepts. The left and right panels of Figure 4 show the mean slope and mean intercept of the power functions that were obtained in Experiment 2 using the same procedure as was used in Experiment 1. The parameters are level and modulus in both panels. A threeway mixed-model ANOVA, in which modulus was a between-subjects factor and level and block were withinsubjects factors, was performed on the slope data. The results showed that the main effect of level was significant $[F(1,18)=8.4, p<.01]$ and that the level $\times$ block interaction was significant $[F(9,162)=4.1, p<.001]$. An analysis of simple main effects revealed that there was a significant effect of block at the low-current level $[F(9,324)=3.5, p<.001]$, but not at the high-current level.

A three-way mixed-model ANOVA also was performed on the intercept data. The results showed that the main effect of modulus was significant $[F(1,18)=23.1, p<$ $.001]$ and that the level $\times$ block interaction was significant $[F(9,162)=8.0, p<.001]$.

\section{Discussion}

The significant level $\times$ block interaction obtained from the magnitude estimates for each modulus group shows that both groups of subjects reported smaller estimates with increasing block for the low-current level and that they kept their estimates constant for the high-current level, independently of block (see Figure 3). The significant level $\times$ block interaction obtained from the intercept data indicates that, at earlier blocks, the intercepts were larger for the low-current level than they were for the high-current level and that, at the later blocks, this relation was reversed (see the right panel of Figure 4). This implies that, for both moduli, the intercept for the low-current level decreased more rapidly than did that for the high-current level. These results are in agreement with those of Experiment 1, but cannot be explained in terms of the regression of magnitude estimates to absolute scale values.

The significant main effect of level obtained from the slope data suggests that slope was larger for the lowcurrent level ( $m=4.1)$ than it was for the high-current level $(m=2.5)$. This result is in agreement with results of Experiment 1. Furthermore, the significant level $x$ block interaction obtained from the slope data indicates that the difference of slopes between the two current levels was small at the first block and that it was larger at the later blocks (see the left panel of Figure 4). This result is in agreement with our previous results (Higashiyama \& Tashiro, 1987), but not in agreement with the results of Experiment 1, in which the slope for the low-current level was shown to fluctuate greatly as a function of block.

\section{EXPERIMENT 3}

In Experiment 3, we further investigated whether sensory adaptation was responsible for the decreases of magnitude estimates that were found for the low-current levels in Experiments 1 and 2. Experiment 3 was a repetition of Experiment 1, except that it consisted of four judgmental and six empty blocks. In the judgmental blocks, which were the first two and last two consecutive blocks (Blocks 


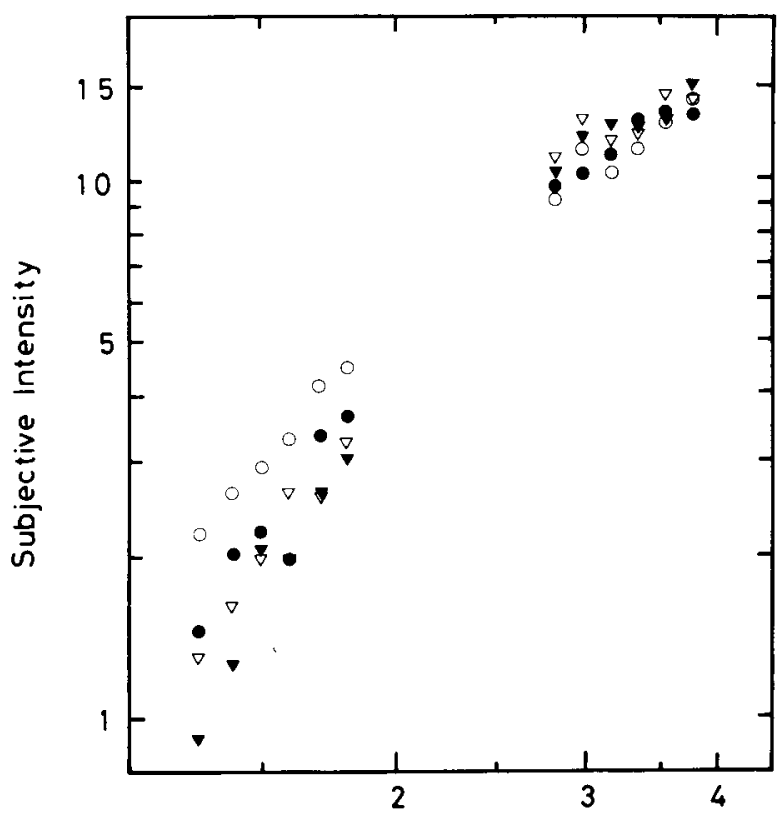

Ratio of Stimulus to Threshold

Figure 5. Log magnitude estimate as a function of $\log$ ratio of stimulus current to threshold current. Each point is the geometric mean of 20 magnitude estimates. The parameter is block $O=1$ st; $\bullet=$ 2nd; $\nabla=9$ th; $\nabla=10$ th. Blocks 3-8 constituted a total-of-8min rest period where the subjects made no judgments.

$1,2,9$, and 10), the subjects made magnitude estimations in accordance with the procedure of Experiment 1. In the empty blocks (Blocks 3-8), no current pulse was delivered on any trial, and the subjects made no estimations during about $8 \mathrm{~min}(6 \times 6 \times 13.5 \mathrm{sec})$. The subjects usually spent the empty trials chatting with the experimenter. Twelve undergraduates were employed as subjects; 7 of these subjects had participated in Experiment 1 or 2.

If sensory adaptation takes place only for repeated stimulation by low current, the estimates or intercept would generally decrease with increasing block, but the estimates and intercept for Block 9 would not be smaller than those for Block 2 , because sensory adaptation would not occur in the absence of stimulation.

\section{Results}

The results of Experiment 3 are given in Figure 5, in which the geometric mean of magnitude estimates is plotted as a function of $\phi / \phi_{0}$, with the parameter of block.

Magnitude estimates. A three-way (level $\times$ stimulus $x$ block) ANOVA for repeated measures was performed on the logarithmic transformations of magnitude estimates. The results showed that main effects of stimulus $[F(5,55)=54.7, p<.001]$ and level $[F(1,11)=24.5$, $p<.001]$ were reliable.

The stimulus $\times$ level interaction was significant $[F(5,55)=14.7, p<.001]$, suggesting that the growth of magnitude estimates was more rapid for the low-current level than it was for the high-current level.

The level $\times$ block interaction also was significant $[F(3,33)=5.5, p<.01]$. An analysis of simple main effects of this interaction indicated that the estimates for the low-current level were smaller for later blocks $[F(3,33)=7.5, p<.001]$, whereas the estimates for the high-current level were constant over blocks. A series of direct comparison tests (Keppel, 1973) for the low-current level showed that the difference between Blocks 1 and 10 was significant $(p<.05)$, but the difference between any other pair of blocks was not.

Slope and intercept. Figure 6 shows the mean slope and mean intercept of the power functions that were obtained in Experiment 3 using the same procedure as was used in Experiment 1. The parameter is the current level. Separate two-way (level $\times$ block) ANOVAs for repeated measures were performed on the slope and intercept data. For the slope data, the main effect of level was significant $[F(1,11)=29.4, p<.001]$. The mean slopes for the high- and low-current levels were .9 and 2.8 , respectively. The level $\times$ block interaction was significant
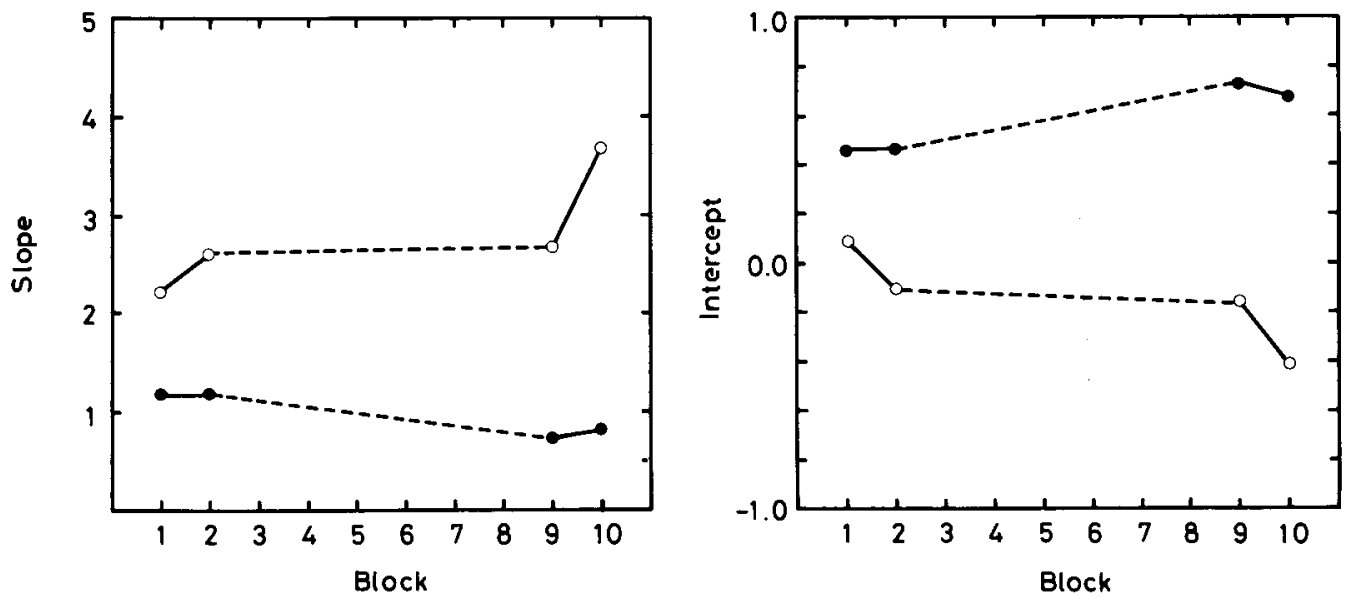

Figure 6. Slope (left) and intercept (right) of power functions fitted to magnitude estimate as a function of block. The parameter is current level. $0=$ low-current level; $\bullet=$ high-current level. 
$[F(3,33)=3.4, p<.05]$. An analysis of simple main effects indicated that the slope for the low-current level increased with increasing block $[F(3,33)=4.8, p<.01]$, but the slope for the high-current level was constant over blocks.

For the intercept data, the main effect of level was significant $[F(1,11)=14.5, p<.001]$. The level $\times$ block interaction also was significant $[F(3,33)=5.0, p<01]$. An analysis of simple main effects of this interaction indicated that the intercept for the low-current level decreased with increasing block $[F(3,33)=4.8, p<$ $.01]$, but the intercept for the high-current level was constant over blocks. A series of direct comparison tests for the low-current level showed that there were significant differences between Blocks 1 and $9(p<.05)$ and between Blocks 1 and $10(p<05)$, but the difference between any other pair of blocks was not significant.

\section{Discussion}

The slopes for the low-current level ( $m=2.8$ ) were consistently larger than were those for the high-current level $(m=1.0)$. This is in agreement with the results of Experiments 1 and 2.

The significant level $\times$ block interaction from the slope data suggests that the difference of slope between the current levels was larger at the later blocks. This is in agreement with the results of Experiment 2.

The most important finding obtained in Experiment 3 is that the estimates (and intercept) for the low-current level generally were smaller for the later blocks, but the difference of estimates (and intercept) between Blocks 2 and 9 was not significant. This finding implies that the adaptation process stopped during the rest period. Furthermore, the finding that the intercept for Block 9 was smaller than was that for Block 1 implies that recovery from the adaptation process was not complete after the rest period. In any event, these findings are not contrary to the hypothesis that sensory adaptation progresses by repeated stimulation of low current.

\section{EXPERIMENT 4}

The purpose of Experiment 4 was to test whether, when judging over the whole current range, the subjects would demonstrate the same outcomes as those obtained in Experiments 1 and 2 . The subjects made magnitude estimations without modulus and standard. Twenty undergraduates were employed as subjects; 11 of these subjects had participated in Experiment 1, 2, or 3.

On the basis of the results of Experiments 1 and 2, it was expected that (1) the trend of decreasing magnitude estimates with increasing block would take place only at the lower current values, (2) obtained exponents would be larger for the low-current than they would be for the high-current level, and (3) a power function might not be appropriate to describe the magnitude estimates for the whole current range, because the exponent depends on current level.

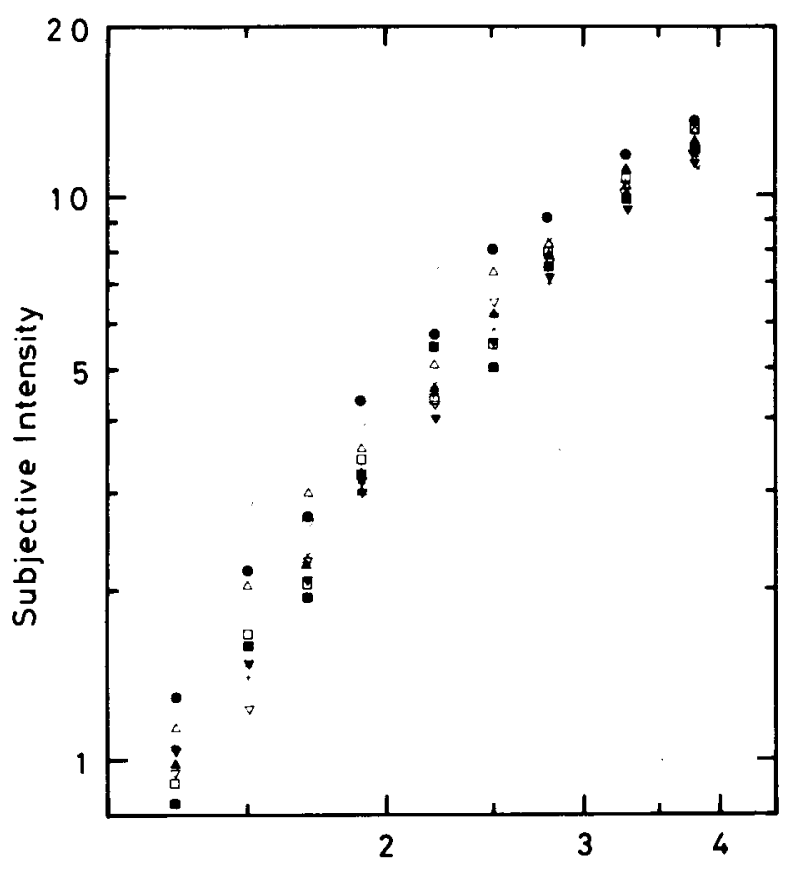

Ratio of Stimulus to Threshold

Figure 7. Log magnitude estimate as a function of $\log$ ratio of stimulus current to threshold current. Each point is the geometric mean of 20 magnitude estimates. The parameter is block: $0=1$ st; $\bullet=$ 2nd; $\Delta=3 \mathrm{rd}$; $\Delta$ = 4th; $\square=$ 5th; $\square=6$ th; $+=7$ th; $x=$ 8th; $\nabla=$ 9th; $\nabla=10 t h$.

\section{Results}

The results of Experiment 4 are given in Figure 7, in which the geometric mean of magnitude estimates is plotted as a function of $\phi / \phi_{0}$, with the parameter of block.

Magnitude estimates. A two-way (stimulus $\times$ block) ANOVA for repeated measures was performed on the logarithmic transformations of magnitude estimates. The results showed that there were significant main effects of stimulus $[F(8,152)=108.3, p<.001]$ and block $[F(9,171)=9.3, p<.001]$, but their interaction was not significant.

Slope and intercepts. For each block, separate power functions were fitted individually to the magnitude estimates for the five lower current values $\left(\phi / \phi_{0}=1.3\right.$ to 2.2 ) and to the magnitude estimates for the five upper current values $\left(\phi / \phi_{0}=2.2\right.$ to 3.8$)$. The magnitude estimates for the central current value were available for either power function. The left and right panels of Figure 8 show mean slope and mean intercept as a function of block with the parameter of current level, respectively. A two-way (level $\times$ block) ANOVA performed on the slope data showed that the main effects of level $[F(1,19)=10.8$, $p<.001]$ and block $[F(9,171)=2.2, p<.05]$ were reliable. An analysis of trend for the main effect of block shows that the quadratic component was significant $[F(1,19)=9.6, p<.001]$.

A two-way ANOVA performed on the intercept data showed that the main effects of level $[F(1,19)=10.5$, 

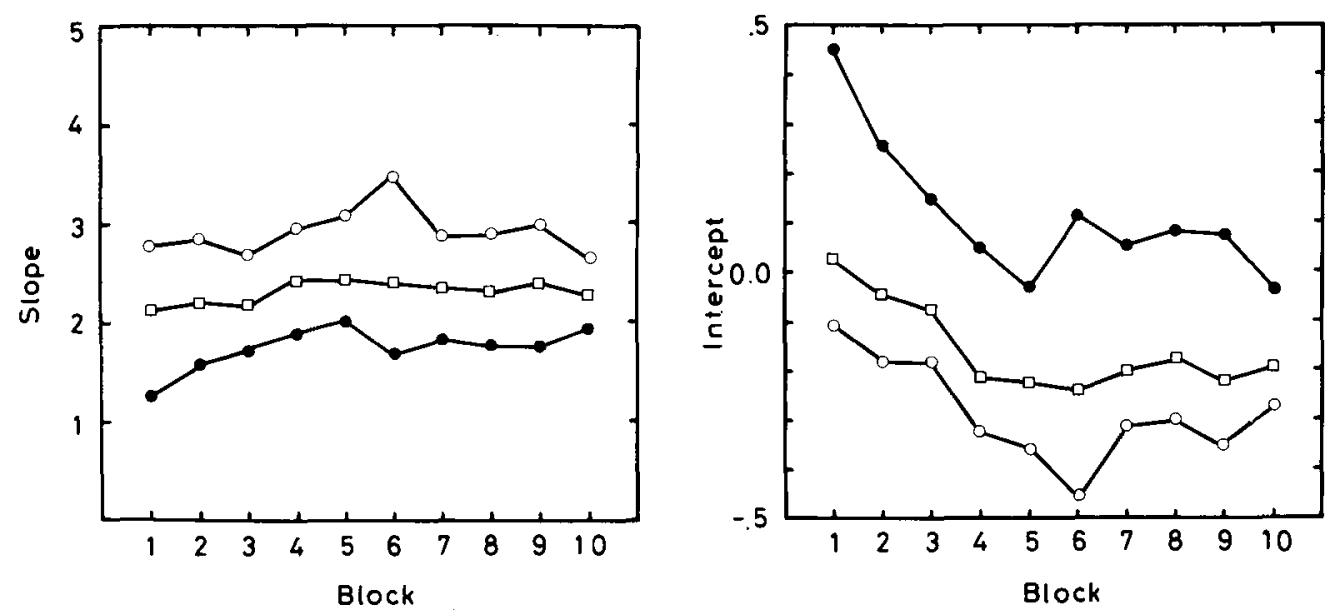

Figure 8. Slope (left) and intercept (right) of power functions fitted to magnitude estimate as a function of block. The parameter is current level: The open circles stand for the low-current level, the filled circles for the high-current level, and the open squares for the whole current range.

$p<.001]$ and block $[F(9,171)=6.0, p<.001]$ were significant. An analysis of trend for the main effect of block showed that the linear $[F(1,19)=11.6, p<.001]$, quadratic $[F(1,19)=8.4, p<.001]$, and cubic $[F(1,19)$ $=8.6, p<.001]$ components accounted for $94 \%$ of the variance.

Power functions were fitted to the individual magnitude estimates for the whole current range. The mean slope and mean intercept are shown in Figure 8 to facilitate comparison with the results from the partial ranges.

A linear function $\left[\psi=a\left(\phi / \phi_{0}\right)+b\right]$ and a logarithmic function $\left[\psi=a \log \left(\phi / \phi_{0}\right)+b\right]$, as well as a power function, were fitted to the same individual magnitude estimates for the whole current range. A comparison of the three functions with respect to coefficient of determination showed that 4 of the 20 subjects produced the highest coefficient for the power function, 9 subjects for the logarithmic function, and 7 subjects for the linear function.

\section{Discussion}

The slope of the power function was larger for the lowcurrent level $(m=2.9)$ than was that for the high-current level $(m=1.7)$. The slope for the whole current range $(m=2.3)$ was intermediate between the extremes. This suggests that the magnitude estimates for the whole current range were concave downward in log-log coordinates. In fact, a comparison of linear, logarithmic, and power functions showed that the power function did not give the best fit for most of the subjects. These findings are in agreement with the results of Experiments 1-3.

Beck and Rosner (1968) and Rosner and Goff (1967) also found that subjective estimates of electrical stimulation were concave downward in $\log$-log coordinates. To describe their data, Rosner and Goff (1967) used a doublelimbed simple power function, as we used in this study. Beck and Rosner (1968), however, used a power function with a threshold correction. Both functions, accounting for more than $95 \%$ of the total variance, were good description of the same data. However, it seems that describing the data in terms of a single smooth function has the disadvantage that it might lead us to think that a single common mechanism determines subjective intensity over the whole current range. The data obtained in Experiments 1-3 suggest two neural mechanisms with different adaptation processes.

The trend of decreasing magnitude estimates with increasing block, however, took place at high- and lowcurrent levels. This seems to contradict the results of Experiments $1-3$, which showed this trend only at the lowcurrent level. It is possible, however, that sensory adaptation occurs for any current range that includes the lowcurrent level. In other words, when high current is repeatedly delivered together with low current in the same session, sensory adaptation may extend to the high-current level and consequently reduce the magnitude estimates over the whole range.

\section{EXPERIMENT 5}

On the basis of the results of Experiments 1-4, it is clear that the mean slope of the power functions fitted to the magnitude estimates was significantly different between the high- and low-current levels. We interpret this difference as a manifestation of two different neural mechanisms. However, one may argue that the difference is attributable to the narrow dynamic range and the subjects' self-imposed limits on response numbers. In other words, the flatter slopes obtained for the high-current level might have been caused by ceiling effects for current close to the maximum presentable and by the subjects' reluctance to use large numbers.

In Experiment 5, our goal was to rule out the explanation of these judgmental biases by constructing a psychophysical scale for apparent distance in an outdoor setting. Apparent distance in outdoor settings is not adaptive for repeated stimulation and is reasonably described as a power function of physical distance (see review by Da Silva, 1985). The distances to be judged ranged from 


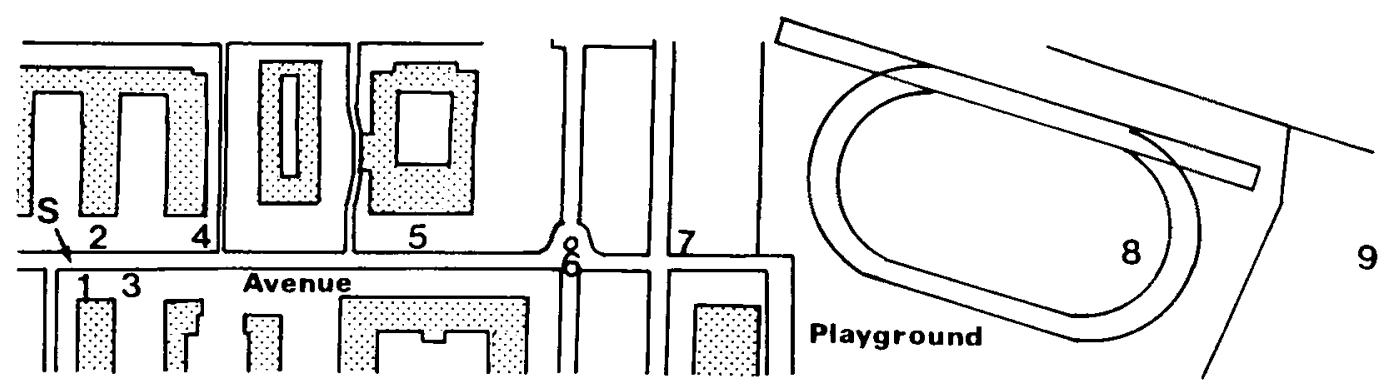

Figure 9. Schematic top view of the test field in Experiment 5. The subject (S) viewed nine targets: $1=$ a sycamore; 2 = a street lamp; 3 = a sycamore; 4 =a telephone booth; 5 = an information board; 6 = a stone monument; $7=\mathrm{a}$ fence; 8 = a soccer goal; $9=$ crematory tower. Shaded portions represent buildings. Targets $1-6$ faced an avenue with roadside trees, Targets 7 and 8 were in the playground, and Target 9 was outside the campus.

5 to $618 \mathrm{~m}$. This range included the greatest distance that could be presented on the university campus.

If the effects found for distance were the same as those found for electrical pulses, these effects would then be attributable to processes of psychophysical judgments. On the other hand, if the slope and intercept of the power function for apparent distance were independent of distance, the results of Experiments 1-4 would reflect somatosensory responses specific to electrical pulses.

\section{Method}

Figure 9 shows a schematic top view of the university campus where we performed Experiment 5 . The subject stood at the end of an avenue ( $8 \mathrm{~m}$ wide $\times 200 \mathrm{~m}$ long) on the campus. From this position, he/she looked at the roadside trees, beyond which lay a playground stretching away through a barricade and a soccer goal to a crematory tower, against the background of the sky. Nine targets were chosen from among various objects scattered between the tree closest to the subject and the crematory tower: a sycamore at a distance of $5 \mathrm{~m}$; a street lamp, $11.5 \mathrm{~m}$; a sycamore, $29 \mathrm{~m}$; a telephone booth, $65 \mathrm{~m}$; an information board, $135 \mathrm{~m}$; a stone monument, $233 \mathrm{~m}$; a fence, $283 \mathrm{~m}$; a soccer goal, $513 \mathrm{~m}$; and the crematory tower, $618 \mathrm{~m}$. These targets were diverse in size, color, and shape.

Twenty subjects made free-modulus magnitude estimations for the apparent distances of the nine targets. Each subject provided 10 estimates for each target. The presentation order of targets was randomized for each subject, with the restriction that a block contained the nine different targets. The experiment was performed under good weather conditions in the fall of 1987.

\section{Results}

Magnitude estimates. The obtained magnitude estimates of Experiment 5 were analyzed in the same way as were those of Experiment 4. Figure 10 shows the geometric mean of magnitude estimates as a function of log physical distance, with the parameter of block. A twoway (distance $x$ block) ANOVA showed that the main effect of distance was significant $[F(8,152)=364.3, p<$ $.001]$ and that the main effect of block and the distance $x$ block interaction were not significant.

Slope and intercept. For each block, separate power functions were fitted individually to the magnitude estimates for the five shorter and the five greater distances. Figure 11 shows the mean slope and mean intercept as a function of block, with the parameter of distance level. A two-way (level $\times$ block) ANOVA on the slope data showed that the block $\times$ level interaction was significant $[F(9,171)=2.3, p<.5]$, indicating that the linear component was significant $[F(1,38)=5.9, p<.05]$.

A two-way ANOVA on the intercept data showed that the block $\times$ level interaction was significant $[F(9,171)$ $=2.2, p<.05]$. An analysis of the trend of this interaction showed the linear $[F(1,19)=4.6, .05<p<.10]$, quadratic $(F<1)$, and cubic $(F<1)$ components were not significant, but the linear component accounted for $61 \%$ of the variance.

Linear, logarithmic, and power functions were fitted to the same individual magnitude estimates for the whole distance range. A comparison of the three functions with respect to coefficient of determination showed that 19 of

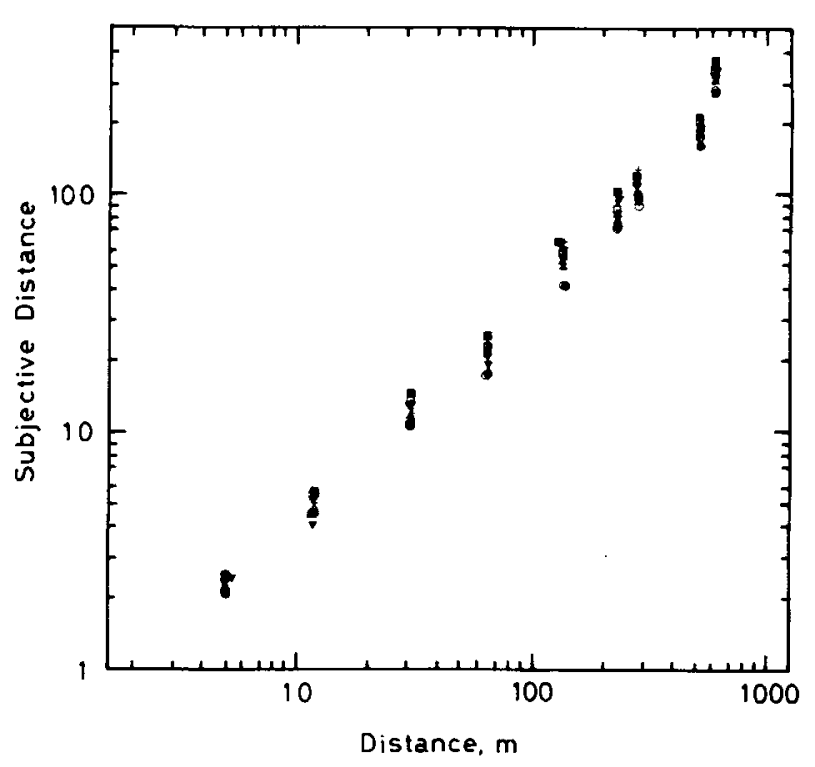

Figure 10. $\log$ magnitude estimate as a function of $\log$ physical distance in meters $(\mathbf{m})$. Each point is the geometric mean of 20 magnitude estimates. The parameter is block: $0=1$ st; $\bullet=2 \mathrm{nd} ; \Delta=$ 3rd; $\Delta=4$ th; $\square=5$ th; $\square=6$ th; $+=7$ th; $x=8$ th; $\nabla=9$ th; $\nabla=10$ th. 

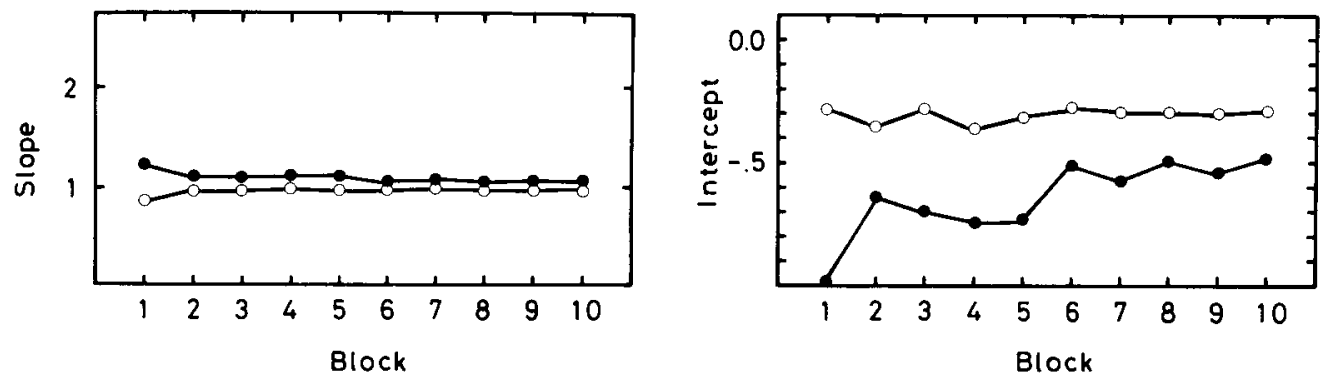

Figure 11. Slope (left) and intercept (right) of power functions fitted to magnitude estimate as a function of block. The parameter is the distance level. $\circ=$ short-distance level; $\bullet=$ great-distance level.

the 20 subjects produced the highest coefficient for the power function, 1 subject for the linear function, and no subjects for the logarithmic function.

\section{Discussion}

The slope of the power function for apparent distance was generally constant ( $m=1.02$ ), independent of block and distance level, although at the first block, the slope for the great-distance level ( $m=1.22)$ was somewhat larger than was that for the close-distance level $(m=.88)$. This implies that the magnitude estimates for the whole distance range are reasonably described by a single power function. For most of the subjects, the power function actually produced a higher coefficient of determination than the linear and logarithmic functions. This is in striking contrast to the results with electrical stimulation.

Sensory adaptation did not occur for apparent distance. The right panel of Figure 11 shows that the difference of intercept between the distance levels was large at the earlier blocks, but, at the later blocks, it was attenuated by the increase of intercept for the great-distance level. This suggests that the magnitude estimates for the greatdistance level rose as a function of block. However, this is not an outcome predicted from current estimates.

It is difficult to suppose that there was a common judgmental process underlying both current and distance estimates. We therefore propose that the results obtained in Experiments 1-4 are specific to electrical stimulation.

Shallower slopes obtained for high-current estimates probably are not attributable to the subjects' reluctance to use large numbers. The subjects did not use numbers greater than 20 , even for extremely heavy shocks; whereas, in distance estimates, they often used numbers greater than 100 for far distances. It therefore appears that the number 20 is not so large as to make a subject reluctant to use it. We think that the subjects, if necessary, could have used larger numbers for electrical shocks.

Table 1

Mean Slopes Obtained for

Each Current Level in Experiments 1-4

\begin{tabular}{lccccc}
\hline & \multicolumn{5}{c}{ Experiments } \\
\cline { 2 - 6 } Level & 1 & 2 & 3 & 4 & Mean \\
\hline Low & 3.9 & 4.1 & 2.8 & 2.9 & 3.4 \\
High & 1.6 & 2.5 & .97 & 1.7 & 1.7 \\
\hline
\end{tabular}

\section{GENERAL DISCUSSION}

\section{Exponent of Power Function}

The most reliable finding obtained in Experiments 1-4 is that the slope of the power function was larger at the low-current level than it was at the high-current level. When low and high currents were judged separately (in Experiments 1-3), the averaged exponents were 3.6 and 1.7 , respectively. When they were judged concurrently (in Experiment 4), the exponents were 2.9 and 1.7. This finding is in agreement with earlier findings by Higashiyama and Tashiro (1987) and Rosner and Goff (1967).

Table 1 shows the mean slopes obtained for each current level in Experiments 1-4. Note that the slope changed greatly from experiment to experiment. For the lowcurrent level, the maximal and minimal slopes were 4.3 and 2.8, respectively; for the high-current level, they were 2.5 and .97 . These findings contrast with the high reliability of slope for other sense modalities. For example, Marks and J. C. Stevens (1966) found that exponent for brightness was very stable (.29 to .35 ) over four experiments, each of which employed a different group of subjects. Wanschura and Dawson (1974) repeated crossmodality matches of loudness to apparent duration (and vise versa) over six sessions. The results showed that duration exponent, for the matches in which time was adjusted, increased from .38 to .45 with session, whereas loudness exponent, for the matches in which loudness was adjusted, remained constant over sessions. Teghtsoonian and Teghtsoonian (1971) found that exponents for apparent length and apparent area were steady over four sessions.

It also should be noted that slope for electrical stimulation was remarkably variable not only over experiments but over blocks within an experiment. In particular, for the low-current level, the slope changed in a complicated manner: In Experiment 1, it fluctuated as a function of block; in Experiments 2 and 3, it increased with increasing block; and, in Experiment 4, it varied curvilinearly, with a maximal value at the intermediate blocks.

Previous studies on electrical stimulation have reported diverse slopes of the power functions fitted to magnitude estimates: Bujas, Szabo, Kovacic, and Rohacek (1975), Hawkes and Warm (1960), J. C. Stevens, Mack, and 

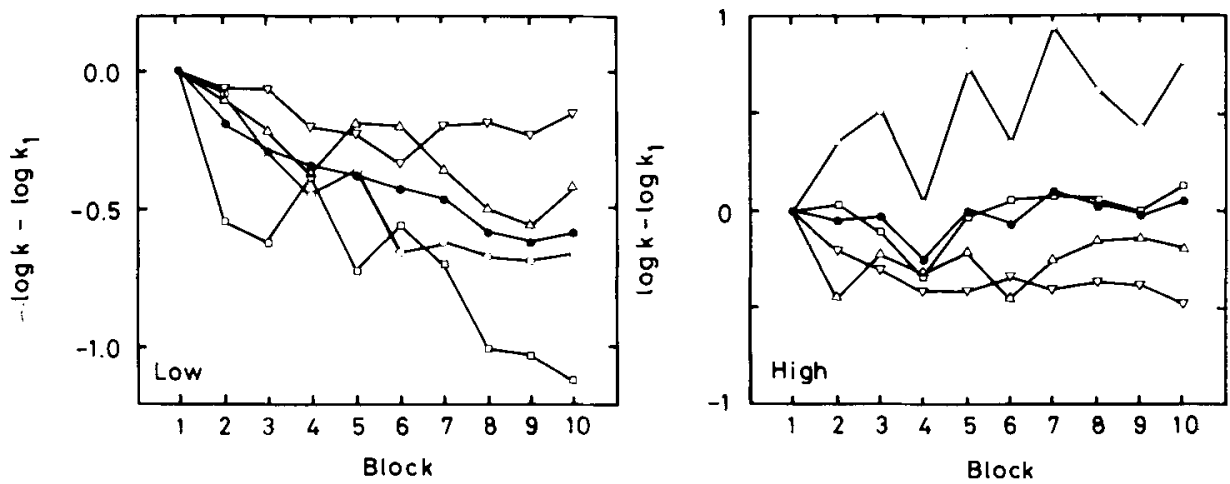

Figure 12. Intercept difference for the low-current (left) and high-current (right) levels as a function of block. The parameter is the modulus: $\Delta=$ free modulus (Experiment 1); $\nabla=$ free modulus (Experiment 4); $0=$ modulus 1 (Experiment 2); $\square=$ modulus 100 (Experiment 2 ); $\bullet=$ means taken across the moduli.

S. S. Stevens (1960), and S. S. Stevens, Carton, and Shickman (1958) obtained high slopes of 3.5 or more; Babkoff (1976, 1978) and Tashiro and Higashiyama (1981) obtained low slopes of .9 to 1.2; Cross, Tursky, and Lodge (1975), Rollman and Harris (1987), and Sachs, Miller, and Grant (1980) obtained moderate slopes of 1.7 to 2.5. Moreover, Rollman and Harris (1987) noted considerable individual differences in the slope of the power function for electrical shock.

This study suggests that such diverse slopes may have been caused mainly by differences in current level. The subjects in the previous studies were often exposed to current pulses determined arbitrarily by the experimenter. In this case, the sensory qualities induced by the particular pulses are usually different for each subject, because individual differences in absolute threshold for electrical pulses are great and its dynamic range is very narrow (Higashiyarna \& Tashiro, 1987; Rollman \& Harris, 1987). Unfortunately, however, since most studies did not report stimulus current relative to threshold current, it may be difficult to guess the sensory qualities induced by the stimulus current.

\section{Adaptation to Electrical Stimulation}

The second important finding of our study is related to adaptation to electrical pulses. When the subjects were exposed separately to the high- and low-current levels, the magnitude estimates for the low-current level generally decreased with increasing block, while those for the high-current level remained constant (Experiments 1-3). On the other hand, when the whole current range was applied in the same session, magnitude estimates for either current level decreased up to the fifth block and fluctuated in subsequent blocks (Experiment 4).

These changes of magnitude estimates with block reflect on the intercept. To normalize the intercept of each block $(\log k)$ relative to that of the first block $\left(\log k_{1}\right)$, the intercept difference $\left(\log k-\log k_{1}\right)$ was obtained for Experiments 1,2 , and 4 . The left panel of Figure 12 shows the intercept difference plotted against block for the lowcurrent level; the right panel for the high-current level. In both panels, the parameter is the modulus employed in the experiments. The filled circles represent the means taken across the modulus conditions.

In Figure 12, it clearly can be seen that the mean intercept difference for low current decreased monotonically with increasing block. The mean intercept difference for the final block amounted to about -.6 , suggesting that current was felt to be one fourth as intense as it had been felt to be at the first block. The mean intercept difference for high current fluctuated around zero as a function of block. This implies that current was felt much the same through the blocks.

Although, to our knowledge, no one has ever pointed out adaptation to electrical pulses, it appears that sensory adaptation to the stimuli near the threshold (or physiological zero) is characteristic of cutaneous systems. For mechanical tactile sensation, Nafe and Wagoner (1941) showed that the lighter the weight of the stimulus, the shorter the time necessary for complete adaptation, that is, complete disappearance of tactile sensation. For warmth and cold, Kenshalo (1970) showed that the adaptation zone, in which perfect adaptation is achieved and the subject reports neither warm nor cold, is approximately $3^{\circ} \mathrm{C}$ on either side of natural physiological zero (usually $29^{\circ}$ to $37^{\circ} \mathrm{C}$ ).

The two findings obtained in this study-dependence of slope and adaptation on sensation level-bring to mind a duplex sensory system of electrocutaneous responses. Recent physiological studies have suggested a close relation between sensory quality and fiber size. For example, Hallin and Torebjörk $(1973,1976)$, reviewing the literature on electrical stimulation of human cutaneous fibers, reported that usual tactile sensations are associated with responses of myelinated large A-alpha fibers and that prick pain and delayed burning pain are activated by myelinated small A-delta fibers and unmyelinated small C fibers, respectively (see also review by Sinclair, 1981).

\section{Judgmental Bias}

Although no effects of modulus on magnitude estimates were found in Experiment 2, they might be suggested in Figure 12. For example, the intercept differences for the 
low-current level produced a negatively steeper slope for modulus 100 than for free modulus. On the other hand, the intercept differences for the high-current level yielded a positively steeper slope for modulus 1 than they did for free modulus. These trends might have been due to regression of magnitude estimates to absolute scale values.

Furthermore, a comparison between Experiments 1 and 4 , in which separate groups of subjects judged current with a free-modulus version of the method of magnitude estimates, suggests that there is a rigid correspondence between numerals and subjective magnitude. In Figures 1 and 7, for example, the number assigned to the current ratio of 1.5 is about 2 and the number assigned to the ratio of 3.0 is about 10 . These results suggest that there is an absolute scale affecting magnitude estimates for electrical shock.

\section{Comparison of Electrical Stimulation With Mechanical Vibration}

Electrocutaneous stimulation of moderate intensity often cannot be distinguished from its mechanical counterpart as a tap and a buzz. Sherrick and Rogers (1966) and von Békésy (1967), noting this similarity between the two types of stimulation, substituted electrical stimulation for the mechanical vibration and taps. Therefore, it might be worthwhile to compare the present results with the properties of mechanical vibrotaction. Verrillo (1974), Verrillo and Chamberlain (1972), and Verrillo, Fraioli, and Smith (1969) investigated subjective magnitude of vibrotaction as a function of skin displacement, with frequency as the parameter. The magnitude estimation and production data were described by a double-limb function on $\log -\log$ coordinates, and the growth of subjective magnitude near the threshold was steeper than was that at suprathreshold. Verrillo, Fraioli, and Smith (1969) found that the steep portion extended for an extremely low frequency. On the basis of these and additional findings, they have proposed that there is a duplex system of cutaneous receptors that make selective responses for high and low frequencies.

However, there is some evidence suggesting that electrocutaneous and mechanical vibrotactions are not attributed to the same sensory system. First, the electrocutaneous system does not respond selectively to frequency. Hahn (1958) showed that absolute threshold for electrical pulses was determined by pulse width, not by frequency. Second, the time necessary for perfect recovery from the adaptation process is shorter for mechanical vibration than it is for electrical stimulation. Our present study showed a recovery duration of more than $8 \mathrm{~min}$ for electrical stimulation, whereas Hahn's $(1966,1968)$ studies showed that it was about $4 \mathrm{~min}$ for mechanical vibration. Thus, it seems that the mechanism of electrocutaneous stimulation differs from that of mechanical vibration, although their perceptual appearances may be similar.

\section{REFERENCES}

BaBkoff, H. (1976). Magnitude estimation of short electrocutaneous pulses. Psychological Research, 39, 39-49.

BABKOFF, H. (1978). Electrocutaneous psychophysical input-output functions and temporal integration. Perception \& Psychophysics, 23, 251-257.

BECK, C., RosNer, B. S. (1968). Magnitude scales and somatic evoked potentials to percutaneous electrical stimulation. Physiology \& Behavior, 3, 947-953.

Bujas, Z., Szaro, S., Kovacic, M., Rohacek, A. (1975). Sensory scales for electrical stimuli in three sense modalities. Acta Instituti Psychologici (University of Zagreb), 75, 17-23.

Cross, D. V., TuRSKY, B., LoDGE, M. (1975). The role of regression and range effects in determination of the power function for electric shock. Perception \& Psychophysics, 18, 9-14.

DA SILVA, J. A. (1985). Scales for perceived egocentric distance in a large open field: Comparison of three psychophysical methods. American Journal of Psychology, 98, 119-144.

Dixon, W. J., \& MASSEY, F. J. (1957). Introduction to statistical analysis. New York: McGraw-Hill.

Gescheider, G. A. (1985). Psychophysics: Method, theory, and application (2nd ed.). Hillsdale, NJ: Erlbaum.

HAHN, J. F. (1958). Cutaneous vibratory thresholds for square-wave electrical pulses. Science, 127, 879-880.

HAHN, J. F. (1966). Vibrotactile adaptation and recovery measured by two methods. Journal of Experimental Psychology, 71, 655-658.

HAHN, J. F. (1968). Low-frequency, vibrotactile adaptation. Journal of Experimental Psychology, 78, 655-659.

Hallin, R. G., \& TOREBJöRK, H. E. (1973). Electrically induced A and $\mathrm{C}$ fibre responses in intact human skin nerves. Experimental Brain Research, 16, 309-320.

Hallin, R. G., \& Torebjörk, H. E. (1976). Studies on cutaneous A and $C$ fiber afferents, skin nerve block and perception. In $Y$. Zotterman (Ed.), Sensory functions of the skin in primates (pp. 137-149). Oxford: Pergamon Press.

HAWKES, G. R., \& WARM, J. S. (1960). Maximum $I_{\mathrm{t}}$ for absolute identification of cutaneous electrical intensity level. Journal of Psychology, 49, 279-288.

HELLSTRÖM, A. (1985). The time-order error and its relatives: Mirrors of cognitive processes in comparing. Psychological Bulletin, 97, 35-61.

Higashiyama, A., \& Tashiro, T. (1983). Temporal and spatial integration for electrocutaneous stimulation. Perception \& Psychophysics, 33, 437-442.

Higashryama, A., \& Tashiro, T. (1987). Magnitude estimates for electrical shock. Japanese Psychological Research, 29, 81-88.

HigashiYama, A., \& TAshiRo, T. (1988). Temporal integration of double electrical pulses. Perception \& Psychophysics, 43, 172-178.

Kenshalo, D. R. (1970). Psychophysical studies of temperature sensitivity. In W. D. Neff (Ed.), Contributions to sensory physiology (Vol. 4, pp. 19-74). New York: Academic Press.

KEPPEL, G., (1973). Design and analysis: A researcher's handbook. Englewood Cliffs, NJ: Prentice-Hall.

MarKs, L. E., \& Stevens, J. C. (1966). Individual brightness functions. Perception \& Psychophysics, 1, 17-24.

NAFE, J. P., \& WAGONER, K. S. (1941). The nature of pressure adaptation. Journal of General Psychology, 25, 323-351.

Rollman, G. B., \& HaRRIs, G. (1987). The detectability, discriminability, and perceived magnitude of painful electrical shock. Perception \& Psychophysics, 42, 257-268.

RosNer, B. S., \& GoFF, W. R. (1967). Electrical responses of the nervous system and subjective scales of intensity. In W. D. Neff (Ed.), Contributions to sensory physiology (Vol. 2, pp. 169-221). New York: Academic Press.

SaChs, R. M., Miller, J. D. , Grant, K. W. (1980). Perceived magnitude of multiple electrocutaneous pulses. Perception \& Psychophysics, 28, 255-262. 
Sherrick, C. E., \& Rogers, R. (1966). Apparent haptic movement Perception \& Psychophysics, 1, 175-180.

SinCLAIR, D. C. (1981). Mechanisms of cutaneous sensations. London: Oxford University Press.

Stevens, J. C., Mack, J. D, \& Stevens, S. S. (1960). Growth of sensation on seven continua as measured by force of handgrip. Journal of Experimental Psychology, 59, 60-67.

Stevens, S. S., Carton, A. S., \&hickman, G. M. (1958). A scale of apparent intensity of electric shock. Journal of Experimental Psychology, 56, 328-334.

Tashiro, T., \& Higashiyama, A. (1981). The perceptual properties of electrocutaneous stimulation: Sensory quality, subjective intensity, and intensity-duration relation. Perception \& Psychophysics, 30, 579-586.

Teghtsoonian, M., \& Teghtsoonian, R. (1971). How repeatable are Stevens's power law exponents for individual subjects? Perception \& Psychophysics, 10, 147-149.

VerRILLo, R. T. (1974). Vibrotactile intensity scaling at several body sites. In F. A. Geldard (Ed.), Cutaneous communication systems and devices. Austin, TX: Psychonomic Society.
Verrillo, R. T., \& Chamberlain, S. C. (1972). The effects of neural density and contactor surround on vibrotactile sensation magnitude. Perception \& Psychophysics, 11, 117-120.

Verrillo, R. T., Fraioli, A. J., \& Smith, R. L. (1969). Sensation magnitude of vibrotactile stimuli. Perception \& Psychophysics, 6, 366-372.

voN BÉkésy, G. (1967). Sensory inhibition. Princeton, NJ: Princeton University Press.

Wanschura, R. G., \& Dawson, W. E. (1974). Regression effect and individual power functions over sessions. Joumal of Experimental Psychology, 102, 806-812.

WARD, L. M. (1973). Repeated magnitude estimations with a variable standard: Sequential effects and other properties. Perception \& Psychophysics, 13, 193-200

ZwisloCki, J. J., \& Goodman, D. A. (1980). Absolute scaling of sensory magnitudes: A validation. Perception \& Psychophysics, 28, 28-38.

(Manuscript received March 14, 1988;

revision accepted for publication December 1, 1988.) 